\title{
Pregnancy and neonatal outcomes after periconceptional exposure to isotretinoin in Koreans
}

Eun-Hwan Cha, MD ${ }^{1,2}$, NaeRy Kim, MD ${ }^{1,3}$, Ho-Seok Kwak, MS', Hae Ji Han, MS', Sung Hong Joo, MD, PhD ${ }^{1,4}$, June-Seek Choi, MD, PhD ${ }^{1,5}$, Kyoung-Chul Chun, MD, PhD², Young-Ah Kim, MD, PhD², Jae-Whoan Koh, MD, PhD'2 Jung Yeol Han, MD, PhD ${ }^{1,2}$

\footnotetext{
${ }^{1}$ Korean Mothersafe Counselling Center, Pregnancy \& Breastfeeding Medicines Information Center, Seoul; Department of Obstetrics and Gynecology, ${ }^{2}$ Inje University Ilsan Paik Hospital, Goyang; ${ }^{3}$ Konkuk University Medical Center, Konkuk University School of Medicine, ${ }^{4}$ National Medical Center,

${ }^{5} \mathrm{CHA}$ Gangnam Medical Center, CHA University, Seoul, Korea
}

\section{Objective}

Isotretinoin should not be used during pregnancy because of the risk of birth defects. Most pregnant women exposed to isotretinoin choose voluntary pregnancy termination due to concerns about birth defects. However, birth outcome data supporting the termination of pregnancy are lacking. This study aimed to evaluate pregnancy and neonatal outcomes after periconception exposure to isotretinoin.

\section{Methods}

This was a prospective cohort study. We evaluated pregnancy and neonatal outcomes after exposure to isotretinoin in 151 pregnant women. Among 1,026 callers at the Korean Teratology Information Service from 2001 to 2017 exposed to isotretinoin during the periconception period, 151 pregnant women who received counseling on teratogenic risk after visiting the clinic were included.

\section{Results}

Among the 151 participants who visited the clinic, only 42 were evaluated using ultrasonography until approximately 20 weeks of gestation. Ultimately, 23 patients were included in the study. The average gestation period during the last exposure to the drug was 2 weeks, and the average daily exposure dose was $12 \mathrm{mg}$. There were two cases of major birth defects in the exposure group. Spontaneous abortion rates were $17.7 \%$ and $8.7 \%$ in the exposure and nonexposure groups, respectively $(P=0.035)$. There was no significant difference between the exposure and non-exposure groups in terms of pregnancy and neonatal outcomes.

\section{Conclusion}

There was no significant difference in pregnancy and neonatal outcomes, including birth defects, between the exposure and non-exposure groups. Further studies with larger sample sizes are required to validate our findings.

Keywords: Isotretinoin; Congenital abnormalities; Teratogens; Pregnancy; Maternal exposure

\section{Introduction}

Isotretinoin (13-cis-retinoic acid), approved by the Food and Drug Administration in 1982, is an effective medication for reducing sebum production, comedogenic acne, and surface and ductal Propionibacterium acnes [1]. In South Korea, it has been approved for the treatment of nodular and cystic acne, not previously treated with conventional treatment [2].

However, isotretinoin has been identified as a teratogenic agent in animal experiments and is classified as a category $X$
Received: 2021.11.25. Revised: 2022.01.05. Accepted: 2022.02.03. Corresponding author: Jung Yeol Han, MD, PhD

Department of Obstetrics and Gynaecology, Inje University Ilsan Paik Hospital, 170 Juhwa-ro, Ilsan-gu, Goyang 10380, Korea E-mail: hanjungyeol055@gmail.com https://orcid.org/0000-0001-5611-2392

Articles published in Obstet Gynecol Sci are open-access, distributed under the terms of the Creative Commons Attribution Non-Commercial License (http://creativecommons org/licenses/by-nc/3.0/) which permits unrestricted non-commercial use, distribution, and reproduction in any medium, provided the original work is properly cited.

Copyright $\odot 2022$ Korean Society of Obstetrics and Gynecology 


\section{Obstetrics \& Gynecology Science}

Eun-Hwan Cha, et al. Periconception exposure to isotretinoin

drug. Exposure to isotretinoin during pregnancy may affect the activity of cephalic neural crest cells, causing craniofacial, cardiac, and thymic malformations. Despite warnings about isotretinoin treatment during pregnancy, malformations have been reported after exposure to isotretinoin.

In 1985, 21 neonatal malformations were reported in 154 pregnant women exposed to isotretinoin in the UK [3]. In a Dutch study conducted in 2014, 51 of 203,962 participants were exposed to isotretinoin from 1999 to 2007, among which $9.4 \%$ reported adverse fetal outcomes. In addition, $50 \%$ of patients chose elective termination of pregnancy [4].

In a study in South Korea, the estimated exposure of pregnant women to isotretinoin was approximately 2.9 per 10,000 births per year, but the incidence is possibly estimated to be higher considering that this study was not a population-based survey [5].

Another Korean study reported that $26.6 \%$ of 79 women exposed to isotretinoin chose voluntary pregnancy termination due to concerns about birth defects, and $50 \%$ of pregnant women exposed to isotretinoin from the last menstrual period (LMP) to 4 weeks after LMP terminated their pregnancy [6].

Several pregnant women have been counseled via telephone or in the clinic after exposure to isotretinoin in the Korean Motherisk Program as a teratology information service

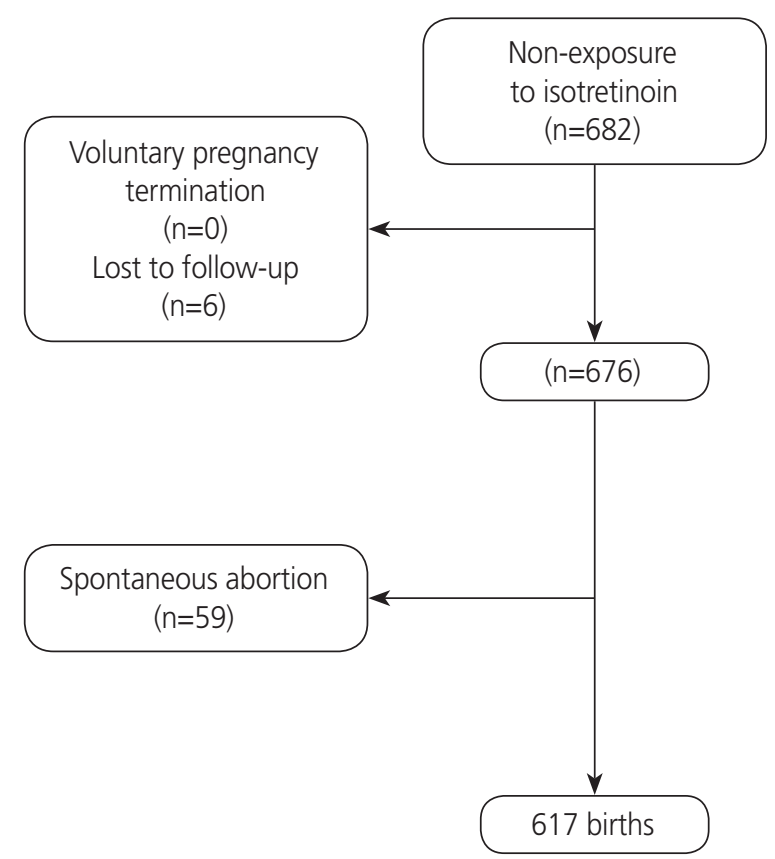

(TIS) supported by the Korean Ministry of Health and Welfare. They need objective and detailed information about the teratogenic risk after exposure to isotretinoin during the periconception period to make an informed decision on whether to continue the pregnancy or terminate it. This study aimed to evaluate pregnancy outcomes after periconception exposure to isotretinoin in Koreans.

\section{Materials and methods}

\section{Data sources and study cohort}

The study was approved by the Institutional Review Board of Cheil General Hospital and the Women's Healthcare Center, Seoul, South Korea. Among 1,026 callers at the Korean TIS from April 2001 to July 2017 exposed to isotretinoin during the periconception period (a critical window that can impact the growth and development of the embryo by the drug), 151 consecutive pregnant women who received counseling on teratogenic risk after visiting the clinic at the Cheil General Hospital and Women's Healthcare Center as part of the Korean Motherisk Program were included in this prospective cohort study. Participants who provided written consent at the time of counseling were recruited (Fig. 1).

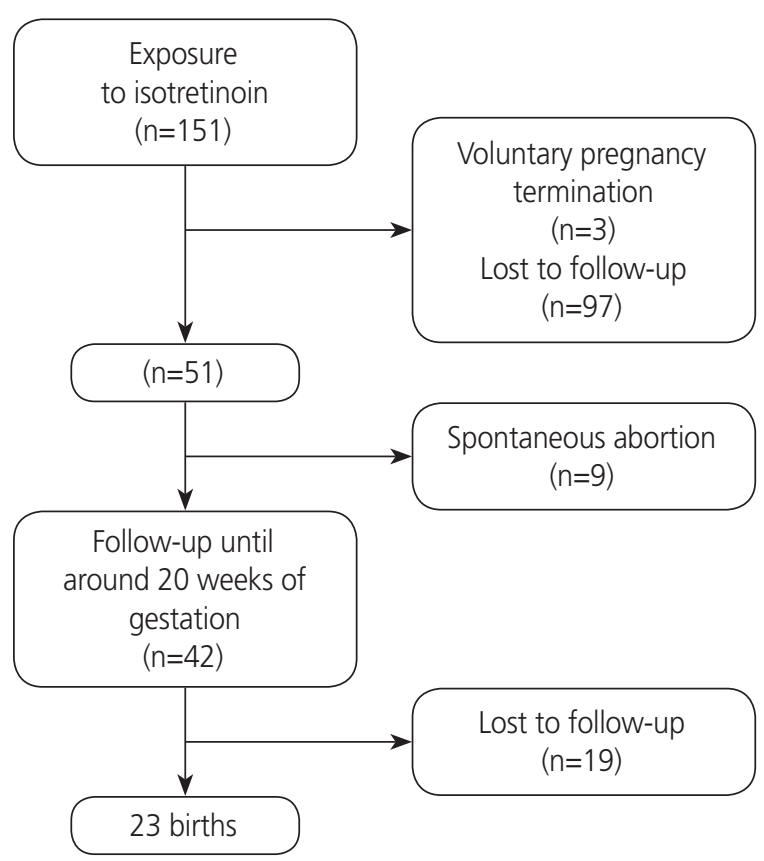

Fig. 1. Study flowchart. 


\title{
Obstetrics \& Gynecology Science
}

\author{
Vol. 65, No. 2, 2022
}

\section{Outcomes}

Data on pregnancy progress and outcomes of the participants were reviewed from the prenatal records, birth records, and neonatal records of the hospital. The outcomes of some participants were determined through telephone interviews. To evaluate fetal gross malformation, ultrasound examinations were performed at approximately 20 weeks of gestational age. An additional level II ultrasound examination was performed if fetal abnormalities were found on ultrasound examination. For neonates born at the hospital, physical examinations were performed by nurses in the delivery room to evaluate gross malformation and neuromuscular abnormalities and by pediatricians in the nursery room to evaluate dermatologic, neurological, cardiovascular, and pulmonary abnormalities. In addition, if abnormalities such as echogenic bowel or choroid plexus cyst were found on prenatal ultrasound examination, additional tests such as abdominal radiography and brain ultrasonography (US) were performed after birth. Malformations were divided into major malformations causing significant functional or cosmetic impairment or being life-limiting and minor malformations that are less significant [7].

In addition, neonatal screening tests for inherited metabolic disorders (such as phenylketonuria, maple syrup urine disease, homocystinuria, hypothyroidism, and hyperadrenocorticism) and hearing difficulty were performed to evaluate adverse events after exposure to isotretinoin. Furthermore, pregnancy and neonatal outcomes were compared between the exposure group and the non-exposure (control) group, which included patients from the same clinic who were not exposed to known teratogenic drugs such as valproate, warfarin, and danazol $(n=617)$.

\section{Statistical analysis}

The chi-square test was used to compare categorical variables, while the Student's $t$-test was used to compare continuous variables. A $P$-value of $<0.05$ was considered statistically significant. SAS software (version 9.4; SAS Institute Inc., Cary, NC, USA) was used for all analyses. Data analyses were performed between April 2001 and July 2017.

\section{Results}

Among the 151 participants, only 42 underwent level II US at the hospital until approximately 20 weeks of gestation. Among the remaining participants, nine reported spontaneous abortions, three underwent voluntary pregnancy

Table 1. Maternal characteristics of the exposure and non-exposure groups

\begin{tabular}{|c|c|c|c|}
\hline Characteristic & Non-exposure group $(n=676)$ & Exposure group $(n=51)$ & $P$-value \\
\hline \multicolumn{4}{|l|}{ General information } \\
\hline Age (yr) & $32.2 \pm 3.8$ & $29.7 \pm 3.8$ & $<0.001$ \\
\hline Gravidity & $2.3 \pm 1.3$ & $1.6 \pm 1.1$ & 0.001 \\
\hline Parity & $0.7 \pm 0.6$ & $0.2 \pm 0.5$ & $<0.001$ \\
\hline Body mass index $\left(\mathrm{kg} / \mathrm{m}^{2}\right)$ & $21.1 \pm 3.7$ & $20.2 \pm 3.1$ & 0.095 \\
\hline \multicolumn{4}{|l|}{ Co-exposure ${ }^{a)}$} \\
\hline Other medications & $682(100.0)$ & $25(59.5)$ & $<0.001$ \\
\hline Alcohol & $274(40.2)$ & $24(57.1)$ & 0.030 \\
\hline Smoking & $46(6.7)$ & $5(11.9)$ & 0.205 \\
\hline Radiation & $121(17.7)$ & $4(9.5)$ & 0.210 \\
\hline \multicolumn{4}{|l|}{ Isotretinoin information ${ }^{b}$} \\
\hline Start exposure in prohibited period (weeks) & - & $-1.0(-4.0$ to 12.0$)$ & - \\
\hline Last exposure in pregnancy (weeks) & - & $2.0(-3.6$ to 13.0$)$ & - \\
\hline Dose/day (mg) & - & $12.0(5.0$ to 20.0$)$ & - \\
\hline Total cumulative dose (mg) & - & $168.3(20.0$ to 600.0$)$ & - \\
\hline
\end{tabular}

Values are presented as mean \pm standard deviation, number (\%), or median (range).

${ }^{a}$ Frequency (percentage of each group); ${ }^{\text {b) }}$ Within 28 days before pregnancy or during pregnancy. 


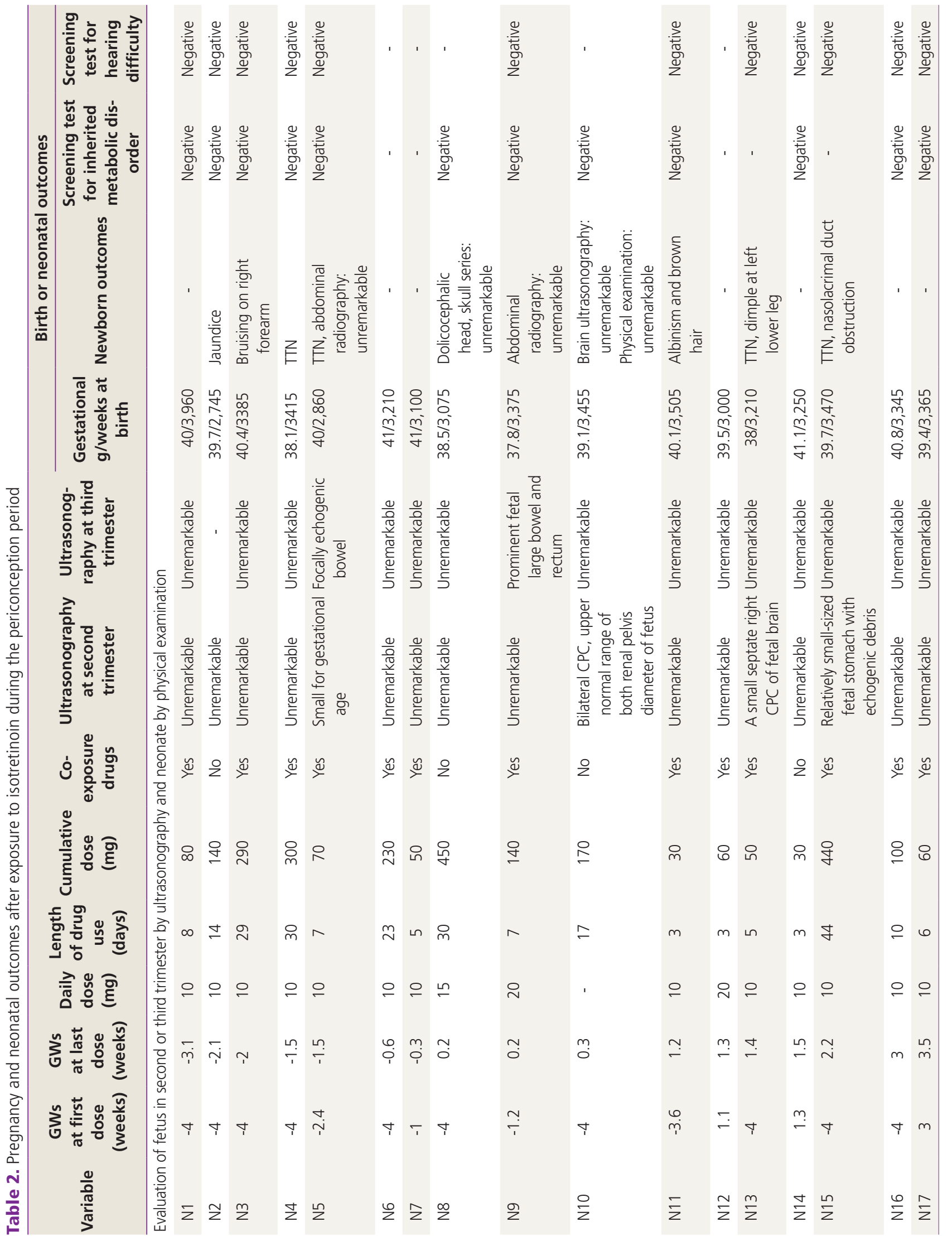




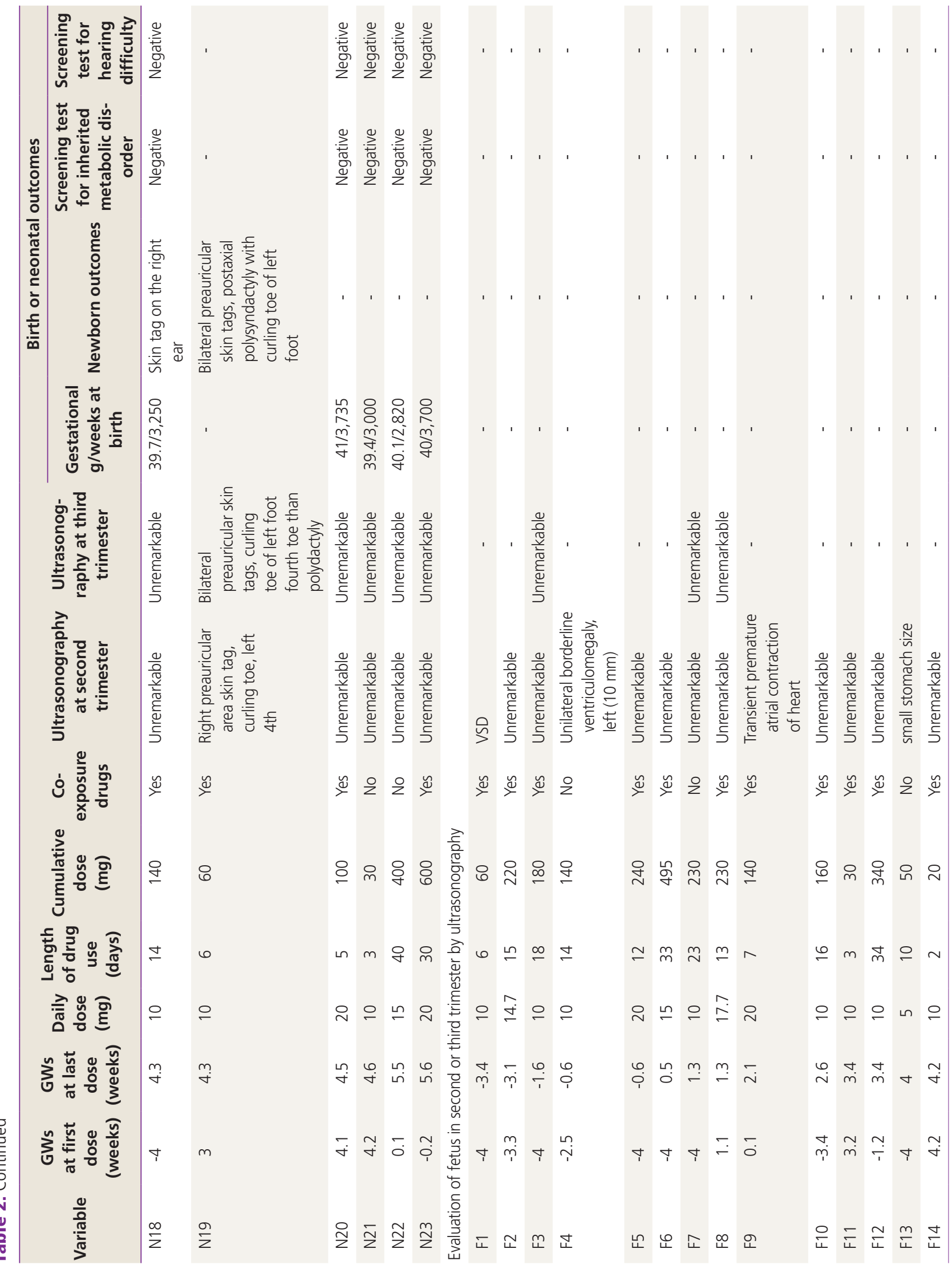




\section{Obstetrics \& Gynecology Science}

Eun-Hwan Cha, et al. Periconception exposure to isotretinoin

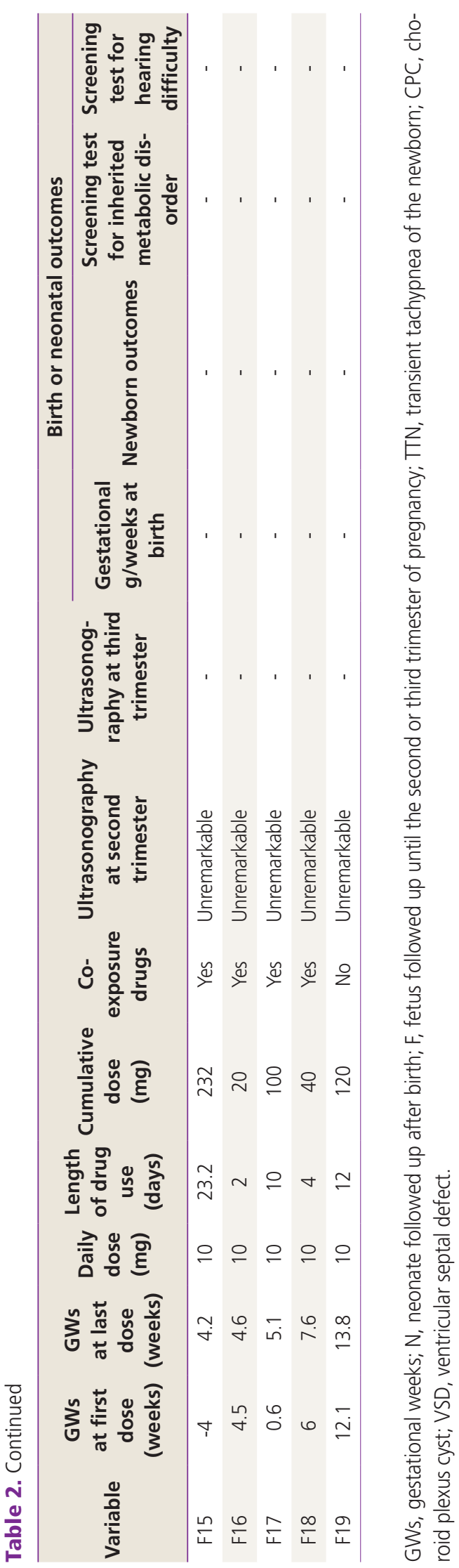

terminations, and 97 were lost to follow-up. Among the 42 pregnant women who underwent level II US, 19 were lost to follow-up after they were transferred to other hospitals. Among the remaining 23 pregnancies, neonatal outcomes were reported from the same hospital and other hospitals in 18 and five pregnancies, respectively. The mean age, gravidity, parity, and the number of other medications used were significantly lower in the exposure group than in the non-exposure group. However, alcohol consumption was significantly higher in the exposure group than in the nonexposure group. The average weeks of gestation during the last exposure of the isotretinoin was 2 weeks (range: from 3.6 weeks before pregnancy to 13.8 weeks of gestation), and the average daily exposure dose was $12 \mathrm{mg}$ (range, 5-20) (Table 1).

Table 2 shows detailed information on exposure of isotretinoin, findings of fetal US, pregnancy, and prenatal outcomes of 42 participants who underwent level II US until approximately 20 weeks of gestation. There was no significant difference between the groups in terms of malformations (Table 3). There were two cases of major birth defects in the exposure group (ventricular septal defect [VSD] and postaxial polysyndactyly with curling toe of the left foot and bilateral preauricular skin tags).

There was no significant difference between the groups in terms of pregnancy and neonatal outcomes. In addition, no abnormal neonatal screening test results for inherited metabolic disorders or abnormal newborn hearing difficulty screening test results were reported after exposure to isotretinoin (Table 4). The rate of spontaneous abortion was $17.7 \%$ $(9 / 51)$ in the exposure group and $8.7 \%$ (59/676) in the nonexposure group (odds ratio, 2.02; 95\% confidence interval, 1.06-3.84; $P=0.035)$.

\section{Discussion}

In this study, we did not find a difference between the exposure and non-exposure groups in terms of pregnancy or neonatal outcomes, including birth defects. However, our data showed that the risk of spontaneous abortion was approximately two times higher in the exposure group than in the non-exposure group.

Dai et al. [8] reported 21 (18\%) cases of spontaneous abortion in 115 prospectively ascertained pregnancies among all 


\section{Obstetrics \& Gynecology Science}

Vol. 65, No. 2, 2022

Table 3. Rate of malformations between the exposure and non-exposure groups

\begin{tabular}{|c|c|c|c|c|}
\hline Variable & $\begin{array}{l}\text { Non-exposure group } \\
(n=676)\end{array}$ & $\begin{array}{l}\text { Exposure group } \\
(n=51)\end{array}$ & $\begin{array}{l}\text { Odds ratio } \\
(95 \% \mathrm{Cl})\end{array}$ & $P$-value ${ }^{\text {a) }}$ \\
\hline Spontaneous abortion & $59(8.7)$ & $9(17.6)$ & $2.022(1.065-3.839)$ & 0.035 \\
\hline Live birth & $617(91.3)$ & $42(82.4)$ & - & - \\
\hline Total malformations & $104(16.9)$ & $7(16.7)$ & $0.989(0.492-1.988)$ & 0.975 \\
\hline Major malformations & $20(3.2)$ & $2^{b)}(4.8)$ & $1.469(0.355-6.075)$ & 0.596 \\
\hline Minor malformations & $84(13.6)$ & $5^{c)}(11.9)$ & $0.874(0.375-2.038)$ & 0.754 \\
\hline
\end{tabular}

Values are presented as number (\%).

$\mathrm{Cl}$, confidence interval.

${ }^{\text {a) }}$ Chi-square test; ${ }^{b}$ Ventricular septal defect $(n=1)$, postaxial polysyndactyly with curling toe of the left foot and bilateral preauricular skin tags $(n=1)$; 'Nasolacrimal duct obstruction $(n=1)$, skin tag in the right ear $(n=1)$, dolichocephalic head $(n=1)$, dimple in the left lower leg $(n=1)$, albinism with brown hair $(n=1)$.

Table 4. Comparison of pregnancy and neonatal outcomes between the exposure and non-exposure groups

\begin{tabular}{lccc}
\hline Variable & Non-exposure group $(\mathbf{n}=\mathbf{6 1 5})$ & Exposure group $(\mathbf{n}=\mathbf{2 3})$ & $\boldsymbol{P}$-value \\
\hline Gestational weeks of birth (weeks) & $39.2 \pm 1.4$ & $39.7 \pm 1.0$ & $0.091^{\mathrm{a})}$ \\
Birth weight $(\mathrm{g})$ & $3,311 \pm 427$ & $3,283 \pm 303$ & $0.761^{\mathrm{a})}$ \\
Height of newborn $(\mathrm{cm})$ & $49.6 \pm 1.8$ & $50.4 \pm 1.3$ & $\left.0.077^{\mathrm{a}}\right)$ \\
Head circumference at birth $(\mathrm{cm})$ & $34.6 \pm 1.2$ & $34.6 \pm 1.1$ & $\left.0.934^{\mathrm{a}}\right)$ \\
Apgar score 1 min & $8.3 \pm 0.7$ & $8.3 \pm 0.6$ & $0.775^{\mathrm{a})}$ \\
Apgar score 5 min & $9.1 \pm 0.6$ & $9.0 \pm 0.5$ & $0.539^{\mathrm{a}}$ \\
NICU admission & $41(6.7)$ & $1(5.6)$ & $1.000^{\mathrm{b}}$ \\
NICU stay days (days) & $12.6 \pm 18.1$ & 8.0 & 0.804
\end{tabular}

Values are presented as mean \pm standard deviation or number (\%).

NICU, neonatal intensive care unit.

${ }^{\text {a) }}$-test; ${ }^{\text {b) }}$ Chi-square test.

reports of isotretinoin-exposed pregnancies that were voluntarily submitted to Hoffmann-La Roche Inc. (Basel, $\mathrm{CH}$ ) in the United States from September 1982 to July 1989. In Canada, there were 1,473 pregnancies at 42 weeks after isotretinoin treatment. Among them, 290 (19.7\%) patients reported spontaneous abortion [9]. In Germany, 22.7\% (5/22) of the patients reported spontaneous abortions among pregnant women exposed to isotretinoin [10]. The rate of spontaneous abortion reported in these studies was similar to that reported in our study. In addition, among 151 pregnant women, we followed up 42 pregnant women exposed to isotretinoin during the periconception period until the second or third trimester of pregnancy. Among the 42 neonates, 23 neonates were followed up. There was no difference in the rates of major birth defects between the groups.

Two cases of major birth defects were reported in the exposure group. VSD was reported in one case exposed to isotretinoin (10 mg/day) from 3.4 to 4 weeks before pregnancy. Postaxial polysyndactyly with a curling toe of the left foot and bilateral preauricular skin tags was reported in another patient exposed to isotretinoin ( $10 \mathrm{mg} /$ day) from 3 to 4.3 weeks of gestation. They might be typical birth defects with retinoid embryopathy, including adverse effects on the brain, face, palate, heart, spinal cord, ear, and thymus, as reported in other studies $[3,8]$.

The teratogenic mechanism of isotretinoin is that it adversely affects the neural crest cells. Treatment with isotretinoin and 4-oxo-isotretinoin caused changes in cytosolic calcium, bleb formation, and cell death in neural crest cells in a previous study [11]. The rates of birth defects due to isotretinoin exposure during pregnancy were 13.6\% (21/154) and $27.7 \%$ (26/94) in the studies by Lammer et al. [3] and Dai et al. [8], respectively.

However, in our study, the rate of major birth defects was 


\section{Obstetrics \& Gynecology Science}

Eun-Hwan Cha, et al. Periconception exposure to isotretinoin

$4.8 \%(2 / 51)$. This rate was similar to that reported by Schaefar et al. [10], who reported one case of a major birth defect (small ventricular septal defect) in 18 live births.

We have recently reported the results of a systematic review and meta-analysis of the rate of major birth defects after isotretinoin exposure during pregnancy; the rate was $15 \%$ in 10 eligible studies including 2,783 women exposed to isotretinoin. Additionally, three studies that included a non-exposed comparison group were eligible for the metaanalysis. The pooled odds ratio for major birth defects in women exposed to isotretinoin was 3.76. After 2006, the pooled odds ratio of major birth defects for isotretinoin exposure was significantly lower (1.04). The odds ratio after 2006 was much lower than that in all the studies. This difference may be explained by the smaller doses and possibly shorter duration of isotretinoin exposure during pregnancy. In terms of exposure dose, the highest dose was up to 87.3 mg/day before 2006 and only 43.7 mg after 2006. Regarding the duration of isotretinoin exposure during pregnancy, the last dose was up to 10 weeks after conception before 2006, while it was 30 days of gestation after 2006 [12]. In our study, the daily exposure dose was only $12 \mathrm{mg}$ (range, 5.0-20.0).

However, our study showed that there are differences in pregnancy and neonatal outcomes between the exposure and non-exposure groups. Published studies on pregnancy or neonatal outcomes including gestational weeks at birth, birth weight, head circumference at birth, Apgar score, inherited metabolic disorder in the newborn, and newborn hearing difficulty screening test results after exposure to isotretinoin during pregnancy are scarce. Loureiro et al. reported birth outcomes in 62 children born to women exposed to topical tretinoin (all-trans-retinoic acid) and 191 children born to unexposed women. The groups were similar in terms of birth weight, height, and head circumference, and there were no significant differences between the groups in terms of gestation length [13].

In South Korea, the incidence of isotretinoin exposure is approximately three pregnancies per 10,000 births [5]. A risk management program (RMP) of isotretinoin as a pregnancy prevention program was launched in June 2019 in South Korea. However, compliance with the RMP was not effective. After the RMP was implemented, the rate of pregnant women exposed to isotretinoin did not decrease. There were 120 pregnant women exposed to isotretinoin at the TIS for 1 year after the RMP.

The hospital compliance on recommendations, including written signature on knowledge as a teratogen, information on effective contraception, and pregnancy test before the prescription was $<50 \%$ [14]. The RMP in South Korea was not as stringent as the iPLEDGE in the USA. The iPLEDGE requires the registration of patient prescribers and pharmacists on a dedicated website and advocates the use of two different types of contraception during isotretinoin treatment and pregnancy tests before prescribing the drug [15]. The RMP should be improved by enhancing the responsibility of clinicians, pharmacists, patients, and manufacturers under the supervision of the Ministry of Food and Drug Safety to protect pregnant women against the risks of the drug and by encouraging wide dissemination of information on the use of isotretinoin during pregnancy.

The strength of this study is that the participants were prospectively followed up to evaluate birth or neonatal outcomes. Furthermore, exposure to isotretinoin during pregnancy and pregnancy and neonatal outcomes were clearly defined without recall bias. Additionally, we evaluated neonatal screening test results for inherited metabolic disorders and newborn hearing difficulty screening test results for exposure to isotretinoin. To the best of our knowledge, this is the first study to evaluate pregnancy and neonatal outcomes after exposure to isotretinoin during the periconception period.

However, in this study, it is not easy to avoid a high probability of a false-negative or type II error because the number of neonates was too small to determine the rate of birth defects. Additionally, our study has some limitations, such as the lack of follow-up of the number of pregnant women and lack of long-term neurodevelopmental outcomes. The outcomes of pregnancy could not be confirmed because 97 of 151 pregnant women exposed to isotretinoin were lost to follow-up after the first-trimester ultrasound examination. The actual occurrence of birth defects is estimated to be higher. In addition, if the neonates did not show abnormal findings on physical examination immediately after delivery, additional follow-up was not performed, and there was no information on long-term neurodevelopment and intellectual ability development.

Despite these limitations, the detailed information on the exposure of isotretinoin and the outcomes of pregnancy and neonates in our study are helpful for pregnant women con- 


\section{Obstetrics \& Gynecology Science}

Vol. 65, No. 2, 2022

sidering whether to continue with pregnancy or terminate it after exposure to isotretinoin during the periconception period.

\section{Conflict of interest}

The authors declare no conflict of interest. The authors alone are responsible for the content and writing of this paper.

\section{Ethical approval}

This study was approved by the Institutional Review Board of Cheil General Hospital and Women's Health Care Center, Seoul, Korea (approval number: CGH-IRB-2010-21).

\section{Patient consent}

Informed consent was not required because this was an analysis of publicly available data.

\section{Funding information}

None.

\section{Acknowledgments}

We thank the counselling team of the Korean Mother Safe Counselling Center for their significant clinical and research contributions.

\section{References}

1. Layton A. The use of isotretinoin in acne. Dermatoendocrinol 2009;1:162-9.

2. Korea Pharmaceutical Information Center. Isotretinoin [Internet]. Seoul (KR): Korea Pharmaceutical Information Center; c2000 [cited 2021 Aug 17]. Available from: http://www.health.kr/searchDrug/result_drug.asp?drug_ $\mathrm{cd}=\mathrm{A} 11 \mathrm{AHHHHH} 0355$.
3. Lammer EJ, Chen DT, Hoar RM, Agnish ND, Benke PJ, Braun JT, et al. Retinoic acid embryopathy. N Engl J Med 1985;313:837-41.

4. Zomerdijk IM, Ruiter R, Houweling LM, Herings RM, Sturkenboom MC, Straus SM, et al. Isotretinoin exposure during pregnancy: a population-based study in the Netherlands. BMJ Open 2014;4:e005602.

5. Kim NR, Yoon SR, Choi JS, Ahn HK, Lee SY, Hong DS, et al. Isotretinoin exposure in pregnant women in Korea. Obstet Gynecol Sci 2018;61:649-54.

6. Yook JH, Han JY, Choi JS, Ahn HK, Lee SW, Kim MY, et al. Pregnancy outcomes and factors associated with voluntary pregnancy termination in women who had been treated for acne with isotretinoin. Clin Toxicol (Phila) 2012;50:896-901.

7. Polyzos KA, Konstantelias AA, Pitsa CE, Falagas ME. Maternal influenza vaccination and risk for congenital malformations: a systematic review and meta-analysis. Obstet Gynecol 2015;126:1075-84.

8. Dai WS, LaBraico JM, Stern RS. Epidemiology of isotretinoin exposure during pregnancy. J Am Acad Dermatol 1992;26:599-606.

9. Henry D, Dormuth C, Winquist B, Carney G, Bugden $S$, Teare $G$, et al. Occurrence of pregnancy and pregnancy outcomes during isotretinoin therapy. CMAJ 2016;188:723-30.

10. Schaefer C, Meister R, Weber-Schoendorfer C. Isotretinoin exposure and pregnancy outcome: an observational study of the berlin institute for clinical teratology and drug risk assessment in pregnancy. Arch Gynecol Obstet 2010;281:221-7.

11. Davis WL, Jacoby BH, Farmer GR, Cooper OJ. Changes in cytosolic calcium, bleb formation, and cell death in neural crest cells treated with isotretinoin and 4-oxo-isotretinoin. J Craniofac Genet Dev Biol 1991;11:105-18.

12. Choi EJ, Kim N, Kwak HS, Han HJ, Chun KC, Kim YA, et al. The rates of major malformations after gestational exposure to isotretinoin: a systematic review and metaanalysis. Obstet Gynecol Sci 2021;64:364-73.

13. Loureiro KD, Kao KK, Jones KL, Alvarado S, Chavez C, Dick $L$, et al. Minor malformations characteristic of the retinoic acid embryopathy and other birth outcomes in children of women exposed to topical tretinoin during early pregnancy. Am J Med Genet A 2005;136:117-21.

14. Choi EJ, Han JY. Non-compliance with pregnancy pre- 


\section{Obstetrics \& Gynecology Science}

Eun-Hwan Cha, et al. Periconception exposure to isotretinoin

vention recommendations for isotretinoin in Korea between 2019-2020. Obstet Gynecol Sci 2021;64:201-8.

15. iPLEDGE. Committed to pregnancy prevention [Inter- net]. Morgantown (NY): iPLEDGE; c2021 [cited 2021

Aug 17]. Available from: https://www.ipledgeprogram. com/\#Main/PublicHame. 